\title{
A MEAN-FIELD MODEL OF SUPERCONDUCTING VORTICES IN THREE DIMENSIONS*
}

\section{S. JONATHAN CHAPMAN ${ }^{\dagger}$}

\begin{abstract}
A mean-field model for the motion of vortices in a type II superconductor is formulated, drawing on analogies with vortices in an inviscid fluid. The model admits discontinuous solutions, and the conditions on such an interface are derived. In a natural limiting case the model is shown to reduce to a novel, vectorial nonlinear diffusion equation. Finally, generalizations of the model to incorporate vortex pinning and fluctuation effects are described.
\end{abstract}

Key words. superconductivity, mean-field, vortices

AMS subject classifications. $82 \mathrm{D} 55$

1. Introduction. The response of a superconducting material to an externally imposed magnetic field is most conveniently described by Fig. 1, which shows the minimum energy state of the superconductor as a function of $H_{0}$, the applied magnetic field, and the material parameter $\kappa$ (known as the Ginzburg-Landau parameter), which determines the type of superconducting material; $\kappa<1 / \sqrt{2}$ describes what is known as a type I superconductor, and $\kappa>1 / \sqrt{2}$ describes what is known as a type II superconductor.

For type I superconductors there is a critical magnetic field $H_{c}$ below which the material will be in the superconducting state but above which it will revert to the normally conducting (normal) state. The transition between normal and superconducting states as the applied magnetic field is raised or lowered through $H_{c}$ takes place by means of phase boundaries separating normal regions from superconducting regions sweeping through the material and is described in [10], [13], and [22].

For type II superconductors a third state exists, which is known as the mixed state. The mixed state, as its name suggests, is neither wholly superconducting nor wholly normal but consists of many normal filaments embedded in a superconducting matrix. Each of these filaments carries with it a quantised amount of magnetic flux and is circled by a vortex of superconducting current; thus these filaments are often known as vortices. The transition from the normal state to the mixed state takes place via a bifurcation as the magnetic field is lowered through some critical value $H_{c_{2}}$ (known as the upper critical field), and is described in [1], [5], [11], [12], [23], [25], and [26]. This bifurcation is subcritical for type I superconductors but supercritical for type II superconductors; hence, although the mixed state exists in type I superconductors, it is unstable and therefore observed only in type II superconductors.

The transition between wholly superconducting and mixed states is studied in [9]. The critical field $H_{c_{1}}$ plotted in Fig. 1 (known as the lower critical field) is calculated on the basis of an energy argument; it is the field at which the energy of the wholly superconducting solution becomes equal to the energy of the single vortex solution for an infinite superconductor. It is found in [9] that there is a barrier to the generation of vortices and that there exists a "superheating field," $H_{s h}$, such that for fields $H_{0}<H_{s h}$ vortices will not be generated even though the wholly superconducting solution may not be the minimum energy solution.

* Received by the editors February 25, 1994; accepted for publication (in revised form) September 6, 1994. This research was supported by a British Nuclear Electric Research fellowship.

$\dagger$ Mathematical Institute, University of Oxford, 24-29 St. Giles', Oxford OX1 3LB, United Kingdom. 


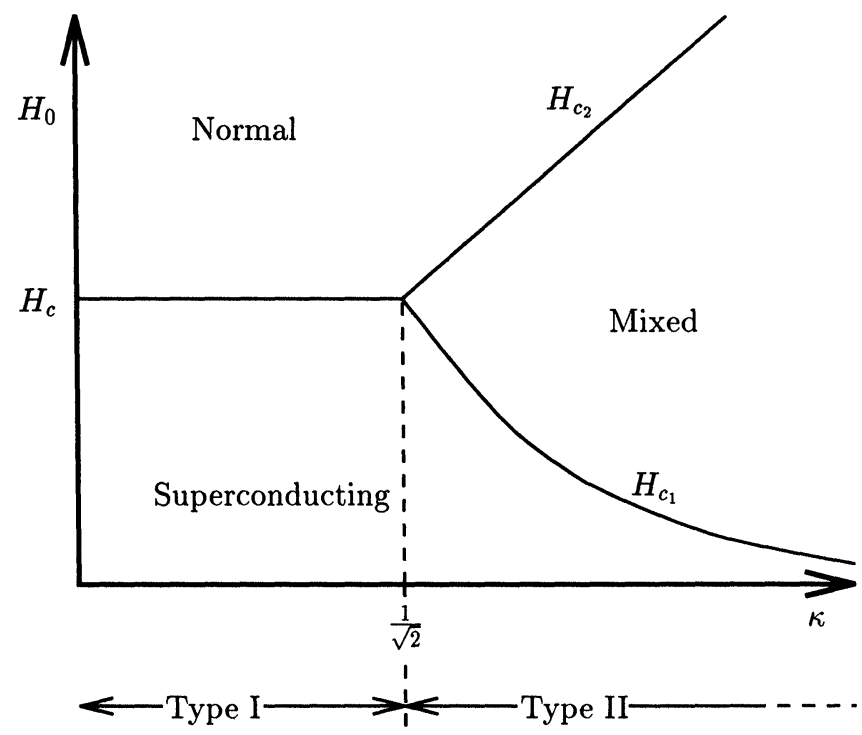

FIG. 1. The response of a superconducting material as a function of the applied magnetic field $H_{0}$ and the Ginzburg-Landau parameter $\kappa$.

In this paper we will consider the behaviour of the superconductor while it is in the mixed state for large values of the parameter $\kappa$ and for fields $H_{0} \ll H_{c_{2}}$.

We will draw heavily on analogies between superconducting vortices and vortices in an inviscid fluid. In an otherwise irrotational, inviscid fluid a (curvilinear) line vortex $\Gamma$ is a solution of the equations

$$
\begin{aligned}
\operatorname{curl} \boldsymbol{u} & =\omega_{0} \delta_{\Gamma}(\boldsymbol{x}) \\
\operatorname{div} \boldsymbol{u} & =0
\end{aligned}
$$

where $\boldsymbol{u}$ is the fluid velocity, $\omega_{0}$ is the circulation of the vortex $\left(\omega_{0}=2 \pi K\right.$, where $K$ is the vortex strength), $\boldsymbol{x}=(x, y, z)$, and

$$
\boldsymbol{\delta}_{\Gamma}(\boldsymbol{x})=\int_{\Gamma} \delta\left(x-x^{\prime}\right) \delta\left(y-y^{\prime}\right) \delta\left(z-z^{\prime}\right) \boldsymbol{d} \boldsymbol{x}^{\prime}
$$

where $\delta$ is the Dirac $\delta$-function. Introducing a vector potential $\boldsymbol{\Omega}$ such that

$$
\begin{aligned}
\operatorname{curl} \boldsymbol{\Omega} & =\boldsymbol{u}, \\
\operatorname{div} \boldsymbol{\Omega} & =0,
\end{aligned}
$$

we find

$$
(\operatorname{curl})^{2} \boldsymbol{\Omega}=-\nabla^{2} \boldsymbol{\Omega}=\omega_{0} \boldsymbol{\delta}_{\Gamma}(\boldsymbol{x}) .
$$

Using the Green's function for the Laplacian on $\mathcal{R}^{3}$ we find that $\Omega$ is given by

$$
\boldsymbol{\Omega}=\frac{\omega_{0}}{4 \pi} \int_{\Gamma} \frac{1}{s} \boldsymbol{d} \boldsymbol{x}^{\prime}
$$

where $s=\boldsymbol{x}-\boldsymbol{x}^{\prime}$ and $s=|\boldsymbol{s}|$. Hence

$$
\boldsymbol{u}(\boldsymbol{x})=\operatorname{curl} \frac{\omega_{0}}{4 \pi} \int_{\Gamma} \frac{1}{s} \boldsymbol{d} \boldsymbol{x}^{\prime}=\frac{\omega_{0}}{4 \pi} \int_{\Gamma} \nabla\left(\frac{1}{s}\right) \wedge \boldsymbol{d} \boldsymbol{x}^{\prime}=-\frac{\omega_{0}}{4 \pi} \int_{\Gamma} \frac{\boldsymbol{s} \wedge \boldsymbol{d}^{\prime}}{s^{3}} .
$$

This is the familiar Biot-Savart law. 
The asymptotic behaviour of (7) as the point $\boldsymbol{x}$ approaches the vortex line is given by $[28]$

$$
\boldsymbol{u} \sim \frac{\omega_{0}}{2 \pi r} \boldsymbol{e}_{\theta}-\frac{\omega_{0}}{4 \pi} \log r C \boldsymbol{b}+O(1)
$$

where $r$ and $\theta$ are local polar coordinates centred on the vortex line, $\boldsymbol{e}_{\theta}$ is the unit vector in the local azimuthal direction, $C$ is the curvature of the vortex line, and $\boldsymbol{b}$ is the unit vector in the binormal direction (see, e.g., [24]).

The law of motion for vortices in an inviscid fluid simply states that they are convected with the fluid velocity. The first term in (8) corresponds to the rotation of the fluid about the vortex line and does not result in any translation. The second term determines the motion of the vortex line and, since it must be evaluated on $r=0$, implies that the vortex moves with infinite velocity in the binormal direction. In actual fact equations (1)-(2) cease to be valid as the vortex core is approached. They are derived as the first term in an expansion of the Navier-Stokes equations as the Reynolds number tends to infinity. Near the vortex core the velocity gradients of this outer solution become unbounded, and an inner region needs to be introduced, in which viscous forces are not negligible. ${ }^{1}$ When the solution in the inner region is matched with the limit of the outer region as the core is approached, the leading-order velocity of the vortex line is found to be

$$
\boldsymbol{v}=-\frac{\omega_{0}}{4 \pi} \log \epsilon C \boldsymbol{b}+O(1)
$$

where $\epsilon$ is the radius of the viscous core region [7].

Let us now turn to superconducting vortices. It has been shown in [14] that as $\kappa \rightarrow \infty$ a superconducting line vortex is a solution of the equations

$$
\begin{aligned}
(\text { curl })^{2} \boldsymbol{H}+\boldsymbol{H} & =2 \pi \boldsymbol{\delta}_{\Gamma}(\boldsymbol{x}) \\
\operatorname{div} \boldsymbol{H} & =0
\end{aligned}
$$

where $\boldsymbol{H}$ is the magnetic field and $\delta_{\Gamma}$ is as before. Equation (10) is known as the London equation. In deriving it in [14] it was assumed that the timescale for the relaxation of the magnetic field was much shorter than the timescale for the motion of vortices, so that the magnetic field is quasi-static. A calculation of these timescales for various materials in [27] indicates that this assumption is valid.

Note the absence of the parameter $\omega_{0}$ in the equations above. This is because, unlike fluid vortices, vortices in a superconductor have quantised strengths. Furthermore, it is found numerically [16], [17] and experimentally [4], [18] that the only stable vortex is that containing exactly one quantum of magnetic flux, and hence all superconducting vortices have the same strength, which we have nondimensionalised to unity.

Using the Green's function for the modified Helmholtz equation on $\mathcal{R}^{3}$ (10) can be inverted to give

$$
\boldsymbol{H}(\boldsymbol{x})=\frac{1}{2} \int_{\Gamma} \frac{e^{-s}}{s} \boldsymbol{d} \boldsymbol{x}^{\prime}
$$

\footnotetext{
1 An alternative regularization involves replacing the vortex by a thin region of distributed vorticity, as in [21], which also results in (9).
} 
where $s$ is as before. The magnetic field $\boldsymbol{H}$ is analogous to the fluid vector potential $\boldsymbol{\Omega}$. The variable analogous to the fluid velocity $\boldsymbol{u}$ is the current $\boldsymbol{j}$, which is given by

$$
\boldsymbol{j}(\boldsymbol{x})=\operatorname{curl} \boldsymbol{H}=\frac{1}{2} \int_{\Gamma} \nabla\left(\frac{e^{-s}}{s}\right) \wedge \boldsymbol{d} \boldsymbol{x}^{\prime}=-\frac{1}{2} \int_{\Gamma} e^{-s}\left(\frac{1}{s^{3}}+\frac{1}{s^{2}}\right) s \wedge \boldsymbol{d} \boldsymbol{x}^{\prime}
$$

Following the argument that led to (8), it is shown in [14] that the asymptotic behaviour of (13) as the point $\boldsymbol{x}$ approaches the vortex core is also given by

$$
\boldsymbol{j} \sim \frac{1}{r} \boldsymbol{e}_{\theta}-\frac{1}{2} \log r C \boldsymbol{b}+O(1)
$$

where $r, \boldsymbol{e}_{\theta}, C$, and $\boldsymbol{b}$ are as before.

The first major difference between fluid vortices and superconducting vortices is in the law of motion. Rather than moving with the current $\boldsymbol{j}$, a superconducting vortex moves in a direction perpendicular to the current, with a velocity given by

$$
v=j \wedge t
$$

where $\boldsymbol{t}$ is the unit tangent to the vortex line. This relationship is often interpreted as follows. The right-hand side can be interpreted as the "Lorentz force" on a vortex line due to the presence of the current, since the vortex carries with it a unit amount of magnetic field. Equation (15) follows from equating this driving force to a ficticious "viscous drag" experienced by the vortex as it moves, which is taken to be proportional to its velocity. (Time has been scaled so that the drag coefficient is unity.)

The asymptotic behaviour (14) would seem to imply that the vortex moves with an infinite velocity in the normal direction. In actual fact, as in the case of a fluid, equations (10)-(11) are simply the outer expansions of a more complicated model as the parameter $\kappa$ tends to infinity. In this case the underlying model is the GinzburgLandau theory. When an inner region is introduced near the vortex core and matched with the limit of the outer region, as in [14], it is found that the leading-order velocity of the vortex is given by

$$
\boldsymbol{v}=-\frac{1}{2} \log \epsilon C \boldsymbol{n}
$$

where $\epsilon$ is again the radius of the vortex core (which is the inverse of the GinzburgLandau parameter $\kappa$ ). It is this asymptotic matching which justifies the relationship (15).

The purpose of this paper is to formulate a mean-field model, in which the individual vortices are homogenized to give a vortex density, or vorticity. We use the case of vortices in an inviscid fluid as a paradigm problem, since there we are already familiar with the mean-field model we hope to obtain -it is simply the Euler equations.

Before we proceed we make a final comment on the laws of motion of vortices. When more than one vortex is present, we may decompose the velocity of a particular vortex into two components. The first is the self-induced velocity, which we denote by $\boldsymbol{v}_{s}$, and the second is the velocity due to all the other vortices present, which we denote by $\boldsymbol{v}_{r e g}$. Using the above arguments, we find that for a fluid

$$
\begin{aligned}
\boldsymbol{v}_{s} & \sim-\frac{\omega_{0}}{4 \pi} \log \epsilon C \boldsymbol{b}+\boldsymbol{O}(1), \\
\boldsymbol{v}_{r e g} & \sim \boldsymbol{u}_{r e g},
\end{aligned}
$$


as $\epsilon \rightarrow 0$, where $\boldsymbol{u}_{\text {reg }}$ is the velocity of the fluid due to all the other vortices (i.e., having removed the singular contribution from the vortex itself). Similarly, for a superconductor

$$
\begin{aligned}
\boldsymbol{v}_{s} & \sim-\frac{1}{2} \log \epsilon C \boldsymbol{n}+\boldsymbol{O}(1), \\
\boldsymbol{v}_{r e g} & \sim \boldsymbol{j}_{r e g} \wedge \boldsymbol{e}
\end{aligned}
$$

where $\boldsymbol{j}_{\text {reg }}$ is the current due to all the other vortices.

Now, $\boldsymbol{u}_{r e g}$ and $\boldsymbol{j}_{\text {reg }}$ will be of order $1 / \delta^{2}$ when the vortices are separated by distances of order $\delta$. For well-separated vortices, that is, for $\delta \sim 1, \boldsymbol{u}_{\text {reg }}$ and $\boldsymbol{j}_{\text {reg }}$ will be $\boldsymbol{O}(1)$ and the leading term in the vortex velocity will be the self-induced motion. Here, however, we consider the limit in which the distance between vortices tends to zero, so than $\delta \ll 1 /|\log \epsilon|^{1 / 2}, \boldsymbol{u}_{\text {reg }}$ and $\boldsymbol{j}_{\text {reg }}$ are very much greater than $\boldsymbol{v}_{s}$, and the self-induced motion is replaced by a motion due to the mean field. For the case of superconducting vortices this argument has been justified in [14], through an asymptotic analysis of densely packed vortices.

2. Paradigm problem: Homogenization of vortices in an inviscid fluid. As a paradigm problem for the homogenization of vortices in a superconductor, we consider the problem of homogenization of line vortices in an inviscid fluid. The model we hope to derive is simply the Euler equations.

We consider the motion of a system of vortices of equal strength. The fluid velocity, $\boldsymbol{u}$, satisfies

$$
\begin{aligned}
\operatorname{curl} \boldsymbol{u} & =\omega_{0} \sum_{i} \delta_{\Gamma_{i}}(\boldsymbol{x}) \\
\operatorname{div} \boldsymbol{u} & =0
\end{aligned}
$$

where the curves $\Gamma_{i}$ are the positions of the vortices and $\omega_{0}$ is the vortex strength. We formally define

$$
\boldsymbol{\omega}_{\eta}(\boldsymbol{x})=\int_{\left|\boldsymbol{x}-\boldsymbol{x}^{\prime}\right|<\eta} \frac{3 \omega_{0}}{4 \pi \eta^{3}} \sum_{i} \boldsymbol{\delta}_{\Gamma_{i}}\left(\boldsymbol{x}^{\prime}\right) d V .
$$

We consider the limit as $\eta \rightarrow 0, \omega_{0} \rightarrow 0$, and the number of vortices tends to infinity (i.e., the separation $\delta \rightarrow 0$ ) in such a way that $\delta \ll \eta$, and $\boldsymbol{\omega}=\lim _{\eta \rightarrow 0} \boldsymbol{\omega}_{\eta}$ is finite. For this to be so we must have $\omega_{0}$ of order $\delta^{2}$, so that $\omega_{0} / \delta^{2}$ is finite. That such a limit exists is the key assumption, and we do not attempt to justify it here. In particular, it is not clear that a distribution of vortices will remain separated by distances of order $\delta$, even if they are so initially. We note that the procedure of averaging discrete line vortices to produce a continuum model is very similar to the vortex method for calculating inviscid, rotational flows, in which continuously distributed vorticity is approximated by point vortices. For two-dimensional flows, in which the vorticity is always directed normal to the plane of interest, the two procedures are effectively inverses, since three-dimensional line vortices reduce to two-dimensional point vortices. In this case, justifying the limiting procedure above is equivalent to proving the convergence of the vortex method, which has been done in [19]. In three dimensions, however, the two procedures differ, since the vortex method uses point vortices, while we are interested here in averaging line vortices. Thus, although the convergence of the point vortex method has been established in [20], the rigorous justification of the limiting procedure above remains open. 
We define the average velocity $\bar{u}$ as

$$
\overline{\boldsymbol{u}}=\lim _{d \rightarrow 0} \frac{3}{4 \pi d^{3}} \int_{\left|\boldsymbol{x}-\boldsymbol{x}^{\prime}\right|<d} \boldsymbol{u} d V,
$$

(where the limiting process is such that $\eta \ll d$ ). It is not difficult to show formally that as $\eta, \delta, d, \omega_{0} \rightarrow 0, \overline{\boldsymbol{u}}$ satisfies

$$
\begin{aligned}
\operatorname{curl} \overline{\boldsymbol{u}} & =\boldsymbol{\omega}, \\
\operatorname{div} \overline{\boldsymbol{u}} & =0 .
\end{aligned}
$$

To close the model we need to derive an evolution equation for $\boldsymbol{\omega}$, which will take the form of a conservation equation.

Suppose the vortices are convected with a velocity field $\boldsymbol{v}$. Consider an arbitrary surface $S$ (not closed), and form a tube $T$ around the vortices which pass through $S$ as shown in Fig. 2. Let $S$ and $T$ be convected with the velocity field $\boldsymbol{v}$. Then no vortices will then enter or leave the tube $T$, since the vortices are convected with the same velocity $\boldsymbol{v}$. Thus the number of vortices crossing $S$ is conserved. This can be written as

$$
\frac{d}{d t} \int_{S} \sum_{i} \boldsymbol{\delta}\left(\boldsymbol{x}-\boldsymbol{x}_{i}\right) \cdot \boldsymbol{n} d S=0
$$

since this integral simply counts the number of vortices crossing the surface $S$. Now as we pass to the limit $\epsilon \rightarrow 0$, it can be shown formally that this integral becomes

$$
\frac{d}{d t} \int_{S} \boldsymbol{\omega} \cdot \boldsymbol{n} d S=0
$$

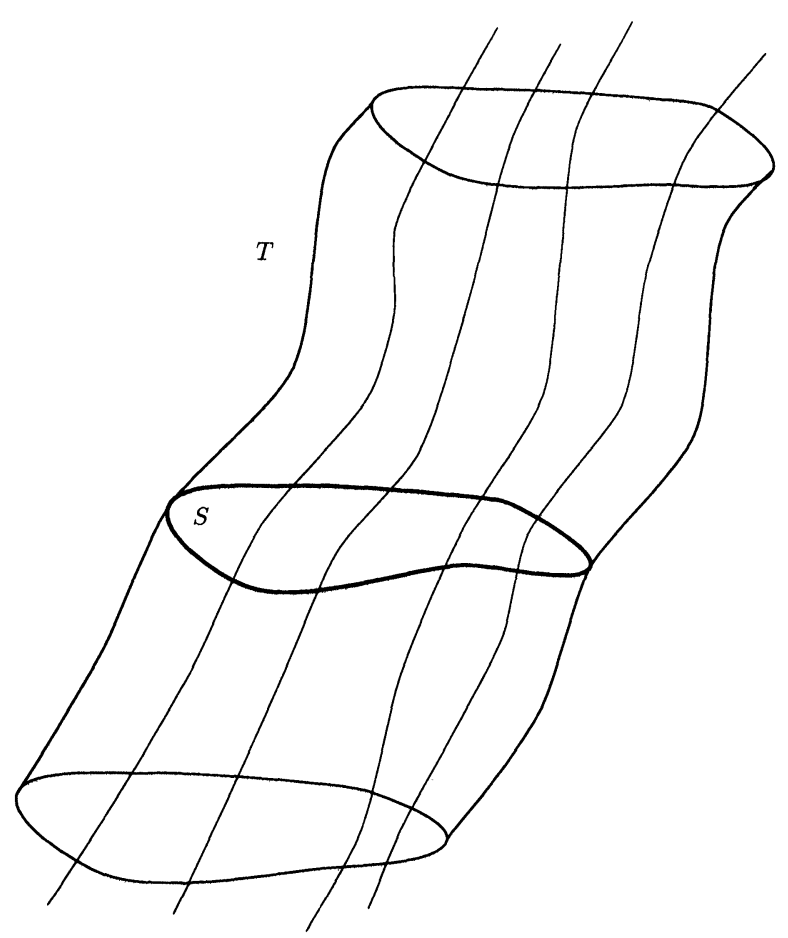

FIG. 2. A tube $T$ enclosing the vortices passing through the surface $S$. 
This is our conservation law in integral form. Since the surface $S$ is arbitrary, we have the equivalent differential form

$$
\boldsymbol{\omega}_{t}+\operatorname{curl}(\boldsymbol{\omega} \wedge \boldsymbol{v})=\mathbf{0} .
$$

It remains to relate the velocity field of the vortices to the velocity field of the fluid. Following the discussion at the end of the introduction, self-induced motion is negligible in comparison to the mean-field motion. Hence the vortices will move with the mean fluid motion, i.e., $\boldsymbol{v}=\overline{\boldsymbol{u}}$.

Thus we arrive at the Euler equations

$$
\begin{aligned}
\boldsymbol{\omega}_{t}+\operatorname{curl}(\boldsymbol{\omega} \wedge \boldsymbol{v}) & =\mathbf{0}, \\
\operatorname{curl} \boldsymbol{v} & =\boldsymbol{\omega}, \\
\nabla \cdot \boldsymbol{v} & =0 .
\end{aligned}
$$

3. A mean-field model of superconducting vortices. We now derive an analagous model for vortices in superconductors. As before we formally define

$$
\boldsymbol{\omega}_{\eta}=\int_{\left|\boldsymbol{x}-\boldsymbol{x}^{\prime}\right|<\eta} \frac{3}{2 \eta^{3}} \sum_{i} \boldsymbol{\delta}\left(\boldsymbol{x}^{\prime}-\boldsymbol{x}_{i}\right) d \boldsymbol{x}^{\prime},
$$

and consider the limit in which $\eta \rightarrow 0$ and the number of vortices tends to infinity (i.e., the separation $\delta \rightarrow 0$ ) in such a way that $\delta \ll \eta$ and the vorticity

$$
\boldsymbol{\omega}=\lim _{\eta \rightarrow 0} \omega_{\eta}
$$

is $O\left(1 / \delta^{2}\right)$. Note that since superconducting vortices all have unit strength we cannot let the strength of the vortices tend to zero as $\delta \rightarrow 0$. The consequence of this is that the magnetic field generated by the vortices is not $O(1)$ but order $1 / \delta^{2}$. However, if we rescale the magnetic field with $1 / \delta^{2}$ the effect is the same as letting the strength of the vortices tend to zero, and it is easy to show formally that as $\eta, \delta, d \rightarrow 0$ in (10), (11) the rescaled average magnetic field $\overline{\boldsymbol{H}}$ satisfies

$$
\begin{aligned}
\overline{\boldsymbol{H}}-\nabla^{2} \overline{\boldsymbol{H}} & =\boldsymbol{\omega}, \\
\nabla \cdot \overline{\boldsymbol{H}} & =0 .
\end{aligned}
$$

As before, we need to write an equation for the evolution of $\boldsymbol{\omega}$ in the form of a conservation law. Given a velocity field $\boldsymbol{v}$ with which the vortices are convected, exactly the same argument holds as in the previous example, and thus we have

$$
\frac{d}{d t} \int_{S} \boldsymbol{\omega} \cdot \boldsymbol{n} d S=0
$$

for an arbitrary surface $S$ convected with the velocity field $\boldsymbol{v}$. As before there is the equivalent differential form

$$
\boldsymbol{\omega}_{t}+\operatorname{curl}(\boldsymbol{\omega} \wedge \boldsymbol{v})=\mathbf{0}
$$

It remains to relate the velocity field $\boldsymbol{v}$ to the magnetic field $\overline{\boldsymbol{H}}$ and the vorticity $\boldsymbol{\omega}$. Following the discussion in the introduction, the self-induced motion is again negligible in comparison to the mean-field motion, so that $\boldsymbol{v}$ is given by

$$
\boldsymbol{v}=\overline{\boldsymbol{j}} \wedge \hat{\boldsymbol{\omega}}=\operatorname{curl} \overline{\boldsymbol{H}} \wedge \hat{\boldsymbol{\omega}}
$$

where $\bar{j}$ is the average current and $\hat{\boldsymbol{\omega}}$ is a unit vector in the direction of $\boldsymbol{\omega}$. 
Thus we arrive finally at the following mean-field model for the motion of vortices in a superconductor (where we drop the overline and use $\boldsymbol{H}$ to denote the average magnetic field):

$$
\begin{aligned}
\boldsymbol{\omega}_{t}+\operatorname{curl}(\boldsymbol{\omega} \wedge \boldsymbol{v}) & =\mathbf{0}, \\
(\operatorname{curl})^{2} \boldsymbol{H}+\boldsymbol{H} & =\boldsymbol{\omega}, \\
\nabla \cdot \boldsymbol{H} & =0, \\
\boldsymbol{v} & =\operatorname{curl} \boldsymbol{H} \wedge \hat{\boldsymbol{\omega}} .
\end{aligned}
$$

Remark. Note that equations (41) and (42) imply that

$$
\nabla \cdot \boldsymbol{\omega}=0
$$

as expected, since vortices cannot start or end except at boundaries. Note also that equation (40) implies $\partial(\nabla \cdot \boldsymbol{\omega}) / \partial t=0$, so that if $\nabla \cdot \boldsymbol{\omega}$ is zero initially it will remain so. Equation (41) then implies that $\nabla \cdot \boldsymbol{H}=0$, so that equation (42) is in fact superfluous in this case.

Remark. The model (40)-(43) for superconducting vortices is ostensibly very similar to the model (30)-(32) for fluid vortices. Indeed, a close inspection reveals only two differences. If the term $\boldsymbol{H}$ were removed from equation (41) and the term $\hat{\boldsymbol{\omega}}$ were removed from equation (43), so that these equations read

$$
\begin{aligned}
(\operatorname{curl})^{2} \boldsymbol{H} & =\boldsymbol{\omega}, \\
\boldsymbol{v} & =\operatorname{curl} \boldsymbol{H}
\end{aligned}
$$

respectively, then $\boldsymbol{H}$ would simply be the vector potential of an inviscid fluid (which reduces to the stream function in two-dimensional flows), and we clearly have $\boldsymbol{\omega}=$ curl $\boldsymbol{v}$ as in equation (31). However, together the two terms omitted change the nature of the equations quite considerably.

Equation (43) describes the nature of the interaction between superconducting vortices. In particular the presence of the term $\hat{\omega}$ means that there is a repulsive/attractive interaction between like/unlike vortices along the line of centers, in contrast to the interaction perpendicular to the line of centres between fluid vortices. This leads to a diffusive type of behaviour and an apparent lack of turbulence among superconducting vortices. For example, a common observation is the formation of a stable two-dimensional hexagonal lattice of superconducting vortices. The corresponding lattice of fluid vortices would be unstable (although von Karmon vortex streets may persist for some time, despite their instability). This difference in the interaction leads us to conjecture that the rigorous justification of the limiting process for superconducting vortices will be easier than the corresponding justification for fluid vortices, since the mean-field model we obtain is "better behaved."

The term $\boldsymbol{H}$ in equation (41) appears at first sight merely to change the decay of the magnetic field $\boldsymbol{H}$ away from regions of vorticity to being exponential rather than algebraic. In fact it has a much greater significance, since it implies that there is a natural lengthscale associated with the model, in contrast to the Euler equations, where lengths can be scaled arbitrarily. We will investigate this aspect of the equations further in $\S 6$.

Remark. It will be useful to calculate the average electric field associated with the model (40)-(43) since this will allow the calculation of effective resistivities due to vortex motion. 
In [14] it is shown that the electric field associated with a single moving vortex is given by

$$
\boldsymbol{E}=\frac{\partial}{\partial t}(\operatorname{curl} \boldsymbol{H})+\boldsymbol{\delta}_{\Gamma} \wedge \boldsymbol{v}
$$

Averaging over many vortices we find

$$
\boldsymbol{E}=\frac{\partial}{\partial t}(\operatorname{curl} \boldsymbol{H})+\boldsymbol{\omega} \wedge \boldsymbol{v}
$$

where $\boldsymbol{E}$ and $\boldsymbol{H}$ now represent the average electric and magnetic fields, respectively. The first term here simply corresponds to the London equation

$$
\boldsymbol{E}=\frac{\partial \boldsymbol{j}_{s}}{\partial t}
$$

while the second term is the electric field generated by the vortex motion.

We note that (46) is consistent with Maxwell's equations, since

$$
\begin{aligned}
\operatorname{curl} \boldsymbol{E}+\frac{\partial \boldsymbol{H}}{\partial t} & =\frac{\partial}{\partial t}\left((\operatorname{curl})^{2} \boldsymbol{H}+\boldsymbol{H}\right)+\operatorname{curl}(\boldsymbol{\omega} \wedge \boldsymbol{v}) \\
& =\frac{\partial \omega}{\partial t}+\operatorname{curl}(\boldsymbol{\omega} \wedge \boldsymbol{v}) \\
& =\mathbf{0}
\end{aligned}
$$

by (40) and (41).

Remark. The model (40)-(43) is only appropriate for a clean homogeneous superconductor, i.e., one with no sites at which vortices are "pinned." In practice, however, it is exactly the pinning of vortices which is the most technologically important aspect of superconductivity. In $\S 7$ we will generalise the model to include such effects. We note here though that even with the introduction of pinning sites, equation (40) will remain valid as before, since pinning will not affect the conservation of vortices. Similarly equation (41) simply describes the magnetic field generated by vortices, which will not be affected by the pinning of vortices. Equation (42) is simply a Maxwell equation. Thus all the extra physics required to model pinning and/or fluctuations must be built into equation (43), which can be seen as a kind of "material law."

Finally we note that, as expected, equations (40)-(43) reduce to the twodimensional equations derived in [15] in the case where $\boldsymbol{H}=(0,0, H(x, y, t)), \boldsymbol{\omega}=$ $(0,0, \omega(x, y, t))$ :

$$
\begin{aligned}
\omega_{t}+\nabla \cdot(\omega \boldsymbol{v}) & =0, \\
-\nabla^{2} H+H & =\omega, \\
\boldsymbol{v}=-\operatorname{sign}(\omega) \nabla H . &
\end{aligned}
$$

4. Boundary conditions. The boundary conditions on the magnetic field $\boldsymbol{H}$ will be the usual boundary conditions at an interface between two media, namely,

$$
\begin{aligned}
{[(1 / \mu) \boldsymbol{H} \wedge \boldsymbol{n}] } & =\mathbf{0} \\
{[\boldsymbol{H} \cdot \boldsymbol{n}] } & =0
\end{aligned}
$$

where [] denotes the jump in the enclosed quantity across $\partial \Omega$ and $\mu$ is the permeability. For most practical applications the permeability of the external region will be close to 
that of the superconducting region, so that these relations simply give the continuity of $\boldsymbol{H}$ across the boundary $\partial \Omega$. These conditions couple equations (40)-(43) to Maxwell's equations in the region external to the superconductor (which we denote by $\Omega_{e}$ ):

$$
\begin{aligned}
\operatorname{curl} \boldsymbol{H} & =\boldsymbol{j} \\
\operatorname{curl} \boldsymbol{E}+\frac{\partial \boldsymbol{H}}{\partial t} & =\mathbf{0} \\
\operatorname{div} \boldsymbol{H} & =0 \\
\operatorname{div} \boldsymbol{E} & =\rho,
\end{aligned}
$$

where $\boldsymbol{E}$ is the electric field and $\rho$ is the charge density. As mentioned earlier, in writing equation (10) we were assuming that the magnetic field relaxes instantly on the timescale for the motion of vortices. In this approximation the normal current flowing in the superconducting material has been neglected in comparison with the superconducting current, except in vortex cores. To be consistent a similar approximation should be adopted in $\Omega_{e}$, giving $\boldsymbol{j}=\mathbf{0}$ there. For most practical applications it will indeed be the case that the current in the external region is negligible in comparison to the current in the superconductor. For the few situations in which it may not be the case the general question of how a normally conducting current becomes a superconducting current as it passes through a metal/superconducting interface needs to be addressed, but this is beyond the scope of this paper.

We now have enough conditions to determine $\boldsymbol{H}$ if $\boldsymbol{\omega}$ is given. The need for additional boundary conditions on $\boldsymbol{\omega}$ depends on whether the characteristics of equation (40) are directed into or out of $\Omega$ on the boundary. If $\boldsymbol{v} \cdot \boldsymbol{n}>0$, where $\boldsymbol{n}$ is the unit outward normal to $\partial \Omega$, the vortices are leaving the sample and no extra information need be given. However, if $\boldsymbol{v} \cdot \boldsymbol{n}<0$, vortices are moving into the sample and we need to give extra information in order to determine the flux of vorticity across $\partial \Omega$.

It is not immediately obvious what this extra boundary condition should be, and this may become the subject of future discussion. We present here one possibility.

It is found experimentally [3], [4] and has been examined theoretically in [9] that no vortices will be generated at the boundary until the current density there reaches a critical value $J_{n u c l}$, which we call the nucleation current density and which is a material parameter which will depend among other things upon surface roughness. Thus if $\mid$ curl $\boldsymbol{H} \mid<J_{n u c l}$ we should have $\boldsymbol{\omega}=\mathbf{0}$, indicating that no vortices are coming in through the boundary.

When the current density is high enough to generate vortices at the boundary, we postulate that the rate of production of vorticity, i.e., the flux of the vorticity through the boundary, will be proportional to the excess of the current density over the nucleation current density. In two dimensions this leads to the condition

$$
-\boldsymbol{v} \cdot \boldsymbol{n} \omega=\alpha\left(|\nabla H|-J_{n u c l}\right)^{+} \quad \text { if } \boldsymbol{v} \cdot \boldsymbol{n} \leq 0,
$$

where $(\cdot)^{+}=\max (\cdot, 0)$. In generalising this equation to three dimensions we need to give also the direction of the flux as well as the magnitude. There are two obvious possibilities: perpendicular to $\boldsymbol{j}$ and parallel to $\boldsymbol{H}$, giving

$$
-\boldsymbol{n} \wedge(\boldsymbol{\omega} \wedge \boldsymbol{v})=\alpha\left(|\boldsymbol{j}|-J_{n u c l}\right)+\frac{\boldsymbol{n} \wedge \boldsymbol{j}}{|\boldsymbol{j}|} \quad \text { if } \boldsymbol{v} \cdot \boldsymbol{n} \leq 0
$$

and

$$
-\boldsymbol{n} \wedge(\boldsymbol{\omega} \wedge \boldsymbol{v})=\alpha\left(|\boldsymbol{j}|-J_{n u c l}\right)+\frac{\boldsymbol{n} \wedge(\boldsymbol{n} \wedge \boldsymbol{H})}{|\boldsymbol{n} \wedge \boldsymbol{H}|} \quad \text { if } \boldsymbol{v} \cdot \boldsymbol{n} \leq 0
$$


respectively. The analysis in [9] indicates that vortices are generated when the electric current density, rather than the magnetic field, becomes too large; thus we would conjecture that the former of these conditions is the more realistic.

In the limit as $\alpha \rightarrow \infty$ the production of vortices when the current density exceeds $J_{n u c l}$ is so rapid that the current density will be immediately reduced to $J_{n u c l}$, and the boundary condition becomes

$\begin{array}{cllll}\text { either } & \boldsymbol{n} \cdot(-\omega \nabla H) & >0 & & \\ \text { or } & \boldsymbol{n} \cdot(-\omega \nabla H)<0 \\ \text { or } & \boldsymbol{n} \cdot(-\omega \nabla H)=0 & \text { and } & |\nabla H|-J_{c}=0 \\ & & \text { and } & |\nabla H|-J_{c}<0\end{array}$

in two dimensions and

$$
\begin{array}{cllll}
\text { either } & \boldsymbol{n} \cdot(\operatorname{curl} \boldsymbol{H} \wedge \boldsymbol{\omega}) & >0 & \\
\text { or } & \boldsymbol{n} \cdot(\operatorname{curl} \boldsymbol{H} \wedge \boldsymbol{\omega}) & <0 \\
\text { or } & \boldsymbol{n} \cdot(\operatorname{curl} \boldsymbol{H} \wedge \boldsymbol{\omega})=0 & \text { and } & |\operatorname{curl} \boldsymbol{H}|-J_{c}=0 \\
& & \text { and } & |\operatorname{curl} \boldsymbol{H}|-J_{c}<0
\end{array}
$$

in three dimensions. However, in taking this limit in three dimensions, we have lost a boundary condition, since (60) is only a scalar equation, while (57) and (58) are vector equations.

We stress that we are only speculating about what may form sensible boundary conditions on (40)-(43). It remains an interesting open problem to determine what type of boundary condition on $\boldsymbol{\omega}$ gives a mathematically well-posed problem. Of course, since our equations are ultimately derived from the Ginzburg-Landau equations, to obtain the boundary conditions on $\boldsymbol{\omega}$ we should really perform an analysis of the rate at which vortices enter a sample using the Ginzburg-Landau model, extending the work in [9].

5. Discontinuous vortex densities. Since equation (40) gives the system (40)-(43) a real characteristic, we have the possibility of solutions with discontinuous $\boldsymbol{\omega}$. Indeed, in two dimensions there is a whole family of static solutions in which $\boldsymbol{\omega}$ is discontinuous with compact support [15].

We now derive the conditions on an interface at which there is a discontinuity in $\boldsymbol{\omega}$. Integrating equation (40) over a small box containing part of the interface gives

$$
v_{n}=\boldsymbol{v} \cdot \boldsymbol{n},
$$

where $v_{n}$ is the normal velocity of the interface. This was to be expected, since if the discontinuity is to propagate it must move at the same speed as the vortices on either side. Applying the same procedure to the equations

$$
\begin{aligned}
\boldsymbol{j} & =\operatorname{curl} \boldsymbol{H} \\
\operatorname{curl} \boldsymbol{j}+\boldsymbol{H} & =\boldsymbol{\omega}
\end{aligned}
$$

leads to the conclusion that

$$
\begin{aligned}
{[\boldsymbol{H} \wedge \boldsymbol{n}] } & =\mathbf{0} \\
{[\operatorname{curl} \boldsymbol{H} \wedge \boldsymbol{n}] } & =\mathbf{0},
\end{aligned}
$$

where [] denotes the jump in the enclosed quantity across the interface. The same argument applied to $\operatorname{div} \boldsymbol{j}=\operatorname{div} \boldsymbol{H}=0$ gives

$$
\begin{aligned}
{[\boldsymbol{H} \cdot \boldsymbol{n}] } & =0 \\
{[\operatorname{curl} \boldsymbol{H} \cdot \boldsymbol{n}] } & =\mathbf{0}
\end{aligned}
$$


while (44) implies

$$
[\boldsymbol{\omega} \cdot \boldsymbol{n}]=0
$$

Hence $\boldsymbol{H}$ and curl $\boldsymbol{H}$ are continuous across the interface. Note that the jump in $\boldsymbol{\omega}$ itself is not determined. Note also that the boundary conditions (61)-(66) are not all independent.

In two dimensions, with $\boldsymbol{H}=(0,0, H(x, y, t)), \boldsymbol{\omega}=(0,0, \omega(x, y, t))$, equations (61)-(63) become

$$
\begin{aligned}
{[H] } & =0, \\
{\left[\frac{\partial H}{\partial n}\right] } & =0, \\
v_{n} & =-\operatorname{sign}(\omega) \frac{\partial H}{\partial n} \quad \text { on } \Gamma,
\end{aligned}
$$

where $v_{n}$ is the normal velocity of the interface $\Gamma$. One case of particular interest is that in which the vorticity is zero on one side of the interface, representing vortices moving into a previously superconducting region. Equations (47)-(49) and (67)-(69) then give

$$
\begin{array}{rlr}
\omega_{t} & =\nabla \cdot(|\omega| \nabla H) \quad \text { in } \Omega_{m}, \\
-\nabla^{2} H+H & =\omega \quad \text { in } \Omega_{m}, & \\
-\nabla^{2} H+H & =0 \quad \text { in } \Omega_{s}, & \\
{[H]} & =0, & \\
{\left[\frac{\partial H}{\partial n}\right]} & =0, & \\
v_{n} & =-\operatorname{sign}(\omega) \frac{\partial H}{\partial n} \quad \text { on } \Gamma,
\end{array}
$$

where $\Omega_{m}$ is the mixed or vortex region and $\Omega_{s}$ is the superconducting region. A travelling wave solution to these equations, representing a wave of vortices moving into a previously superconducting region, has been studied in [8] and was shown to be linearly stable.

6. Nonlinear diffusion as a limiting case. Throughout the analysis so far we have been working on the natural lengthscale for the decay of the magnetic field away from a superconducting vortex, which is known as the penetration depth and denoted by $\lambda_{L}$. We mentioned at the end of $\S 3$ that lengths cannot be scaled arbitrarily in equations (40)-(43). If we scale length instead with a typical length of our superconducting sample, say, $l$ (and rescale time with $l^{2} / \lambda_{L}^{2}$ accordingly), then we arrive at the following equations:

$$
\begin{aligned}
\boldsymbol{\omega}_{t}+\operatorname{curl}(\boldsymbol{\omega} \wedge \boldsymbol{v}) & =0 \\
\boldsymbol{H}-\lambda^{2} \nabla^{2} \boldsymbol{H} & =\boldsymbol{\omega} \\
\nabla \cdot \boldsymbol{H} & =0 \\
\boldsymbol{v} & =\operatorname{curl} \boldsymbol{H} \wedge \hat{\boldsymbol{\omega}}
\end{aligned}
$$

where $\lambda=\lambda_{L} / l$. Typically $\lambda_{L}$ is of the order of tenths of microns, whereas $l$ may be of the order of millimeters. Thus $\lambda$ will be of the order of $10^{-3}-10^{-4}$. If we make the 
assumption that $\boldsymbol{H}$ does not vary too quickly on this lengthscale, so that $\nabla^{2} \boldsymbol{H}$ is not too large, then we may let $\lambda \rightarrow 0$ to obtain the following simplified model:

$$
\begin{aligned}
\boldsymbol{\omega} & =\boldsymbol{H}, \\
\boldsymbol{H}_{t}+\operatorname{curl}(\boldsymbol{H} \wedge(\operatorname{curl} \boldsymbol{H} \wedge \hat{\boldsymbol{H}})) & =0, \\
\nabla \cdot \boldsymbol{H} & =0,
\end{aligned}
$$

where $\hat{\boldsymbol{H}}$ is the unit vector in the direction of $\boldsymbol{H}$. Equation (81) has been previously written by Brandt [6].

Of course, in order to impose boundary conditions on (81) we need to consider boundary layers. When an interface $\Gamma$ between a region in which $\boldsymbol{H}=\mathbf{0}$ and a region in which $\boldsymbol{H} \neq \mathbf{0}$ is examined using inner and outer asymptotic expansions it is found that the free boundary conditions on (81) are

$$
\begin{aligned}
& \boldsymbol{H}=\mathbf{0} \quad \text { on } \Gamma, \\
& v_{n}=\boldsymbol{n} \cdot(\operatorname{curl} \boldsymbol{H} \wedge \hat{\boldsymbol{H}}) \quad \text { on } \Gamma .
\end{aligned}
$$

Equation (81) is a nonlinear diffusion equation and demonstrates the diffusive nature of (40)--(43). The diffusive nature of the equation becomes more obvious if we consider the simpler two-dimensional case in which $\boldsymbol{H}=(0,0, H(x, y, t))$. Then (81) becomes

$$
H_{t}=\nabla \cdot(|H| \nabla H)
$$

which we recognise as a version of the porous medium equation. The boundary conditions (83), (84) become

$$
\begin{aligned}
& H=0 \text { on } \Gamma \text {, } \\
& v_{n}=-\operatorname{sign}(H) \frac{\partial H}{\partial n} \quad \text { on } \Gamma \text {. }
\end{aligned}
$$

There is an extensive literature on equation (85); see [2] for a review.

7. Pinning and fluctuation effects. As we remarked at the end of $\S 3$, the model (40)-(43) is appropriate only for a superconductor which has no sites at which vortices are "pinned." Since most superconducting samples are full of pinning sites (grain boundaries, twin planes, dislocations, impurities, cracks), and since the pinning of vortices is crucial in applications, we generalise the model here to include such effects.

Since equations (40)-(42) hold regardless of pinning or fluctuation effects, all the extra physics needed to model pinning and/or fluctuation effects must be built into equation (43).

Various scales of pinning sites can be introduced. One possible scenario is that of many small pinning sites outnumbering the vortices. In this case it is conjectured that a vortex will not move until the current at that point exceeds a certain critical value, the depinning current $J_{p i n}$, required to break it away from its pinning site. Once moving the direction of the velocity of the vortex will follow the Lorentz force as before, but the presence of a sea of pinning sites will also slow the vortex to beneath its ideal speed. Once the depinning current has been exceeded, the velocity may again be linear in the current density (as in Fig. 3), or it may be that the curve asymptotes 


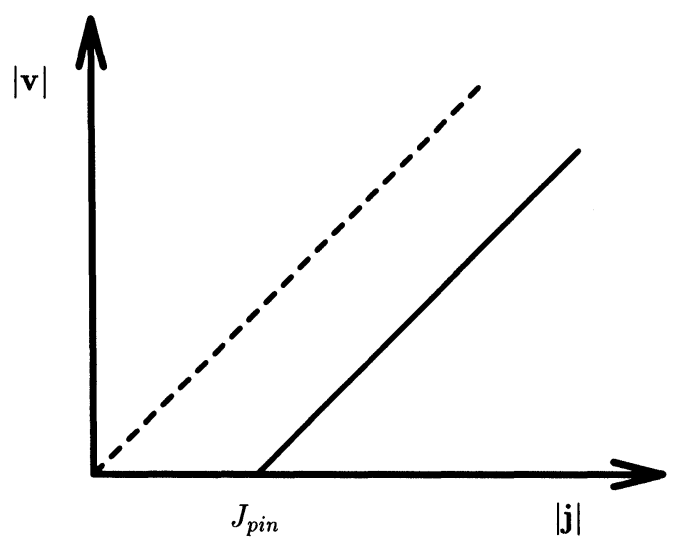

FIG. 3. The possible form of the velocity of a vortex $\boldsymbol{v}$ as a function of the local current density $\boldsymbol{j}$.

to the ideal curve (as in Fig. 4), as the pinning sites have a lesser effect on fast-moving vortices. A careful analysis of the interaction between vortices and pinning sites will be needed to determine this curve theoretically. Of course, it may be determined empirically from experimental measurements of resistivity as a function of current density.

When such a relationship between the vortex velocity and the current is adopted, there will clearly be two regions: one in which $|\boldsymbol{j}|<J_{\text {pin }}$ and the vortices are stationary and one in which $|\boldsymbol{j}|>J_{\text {pin }}$ and the vortices are moving. The result is the following free-boundary problem:

$$
\begin{array}{rlc}
(\text { curl })^{2} \boldsymbol{H}+\boldsymbol{H} & =\boldsymbol{\omega} & \text { in } \Omega_{1}, \\
\boldsymbol{\omega}_{t} & =0 & \text { in } \Omega_{1}, \\
|\operatorname{curl} \boldsymbol{H}| & <J_{\text {pin }} & \text { in } \Omega_{1}, \\
(\operatorname{curl})^{2} \boldsymbol{H}+\boldsymbol{H} & =\boldsymbol{\omega} & \text { in } \Omega_{2}, \\
\boldsymbol{\omega}_{t}+\operatorname{curl}(\boldsymbol{\omega} \wedge \boldsymbol{v}) & =0 & \text { in } \Omega_{2}, \\
|\operatorname{curl} \boldsymbol{H}| & >J_{\text {pin }} & \text { in } \Omega_{2},
\end{array}
$$

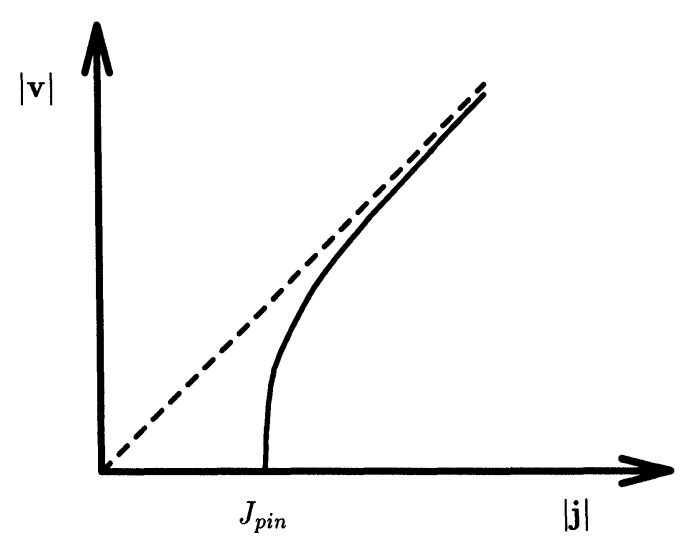

FIG. 4. An alternative possible form of the velocity of a vortex $\boldsymbol{v}$ as a function of the local current density $\boldsymbol{j}$. 


$$
\begin{aligned}
{[\boldsymbol{H}] } & =\mathbf{0}, \\
{[\operatorname{curl} \boldsymbol{H}] } & =\mathbf{0}, \\
|\operatorname{curl} \boldsymbol{H}| & =J_{\text {pin }} \quad \text { on } \Gamma,
\end{aligned}
$$

where $\Gamma$ is the interface between the two regions $\Omega_{1}$ and $\Omega_{2}$, [] represents the jump in the enclosed quantity across $\Gamma$ as before, and

$$
\boldsymbol{v}=(\operatorname{curl} \boldsymbol{H} \wedge \hat{\boldsymbol{\omega}}) \frac{f(|\operatorname{curl} \boldsymbol{H}|)}{|\operatorname{curl} \boldsymbol{H}|}
$$

where $f$ is the function graphed in Fig. 3 or Fig. 4 .

Note that the velocity of the boundary is not given explicitly by the boundary conditions but is implicit. In particular, unlike the interface conditions of $\S 5$, the velocity of the boundary is no longer equal to the local vortex velocity.

If the density of pinning sites is nonuniform throughout the sample, then the depinning current $J_{\text {pin }}$ will be a function of position. It will also be a function of temperature, and thermal effects could be built into the model this way. In particular thermal fluctuations can be modelled by incorporating fluctuations of $J_{p i n}$. It may also be that $J_{p i n}$ is a function of the magnetic field $\boldsymbol{H}$. Further investigation of the nature of pinning is needed to clarify this possibility.

Large-scale pinning sites may be incorporated into the model if we introduce a pinning potential into equation (43), i.e., add an attractive velocity toward certain pinning sites. Some vortices would then be pinned at these sites, and depending on the number of such sites, other vortices may flow around them. Clearly, a combination of both sizes of pinning sites can also be modelled.

8. Conclusion and further remarks. We have derived a mean-field model for the motion of vortices in the mixed state of a type II superconductor. The model consists of a quasi-static equation for the magnetic field coupled with an evolution equation for the vortex density. In a particular limiting case the model reduces to a degenerate, nonlinear diffusion equation, which we considered in $\S 6$. In $\S 7$ we generalised the model to include vortex pinning and fluctuation effects.

Finally, we comment on two further restrictions to the applicability of the model.

Temperature range. Equations (40)-(43) have effectively been derived from the London equation (10) and the Lorentz force law (15). Both of these can be derived as the London limit of the Ginzburg-Landau equations, and therefore the model (40)-(43) should be valid for London superconductors whenever the GinzburgLandau equations are valid.

Hall effect. In the law of motion (15) the velocity of a vortex has been taken to be in a direction perpendicular to the current. In fact there is also a component of the velocity in the direction parallel to the current, due to the Hall effect (by which a moving charged particle is deflected by a magnetic field). This component is generally small in comparison to the Lorentz component (about 10\%) but could easily be included in the law of motion if we simply add another term to (15).

\section{REFERENCES}

[1] A. A. Abrikosov, On the magnetic properties of superconductors of the second group, Soviet Phys. J.E.T.P., 5 (1957), pp. 1174-1182. 
[2] D. G. Aronson, The Porous Medium Equation, Lecture Notes in Mathematics 1224, SpringerVerlag, New York, 1986, pp. 1-46.

[3] R. W. DE Blois And W. DE Sorbo, Surface barrier in type II superconductors, Phys. Rev. Lett., 12 (1964), pp. 499-501.

[4] G. Boato, G. Gallinaro, and C. Rizzuto, Direct evidence for quantized flux threads in type II superconductors, Solid State Comm., 3 (1965), pp. 173-176.

[5] C. Bolley and B. HelfFer, Rigorous Results on Ginzburg-Landau Models in a Film Submitted to an Exterior Parallel Magnetic Field, Ecole Centrale de Nantes, 1994, preprint.

[6] E. H. BRAndT, Flux diffusion in high-T $T_{c}$ superconductors. Z. Phys. B, 80 (1990), pp. 167-175.

[7] A. J. Callegari AND L. Ting, Motion of a curved vortex filament with decaying vortical core and axial velocity, SIAM J. Appl. Math., 35 (1978), pp. 148-175.

[8] S. J. Chapman, Stability of travelling waves in models of superconductivity, IMA J. Appl. Math., (1995), to appear.

[9] —-, Superheating fields of type II superconductors, SIAM J. Appl. Math., 55 (1995), pp. 1233-1258.

[10] - Asymptotic analysis of the Ginzburg-Landau model of superconductivity: Reduction to a free boundary model, Quart. Appl. Math., (1995), to appear.

[11] — Nucleation of superconductivity in decreasing fields I, European J. Appl. Math., 5 (1994), pp. 449-468.

[12] —, Nucleation of superconductivity in decreasing fields II, European J. Appl. Math., 5 (1994), pp. 469-494.

[13] S. J. Chapman, S. D. Howison, And J. R. OCkendon, Macroscopic models of superconductivity, SIAM Rev., 34, 4 (1992), pp. 529-560.

[14] S. J. Chapman and G. Richardson, Motion of vortices in type II superconductors, SIAM J. Appl. Math., 55 (1995), pp. 1275-1296.

[15] S. J. Chapman, J. Rubinstein, and M. Schatzman, A Mean-Field Model of Superconducting Vortices, 1994, preprint.

[16] Q. Du, M. D. Gunzburger, And J. S. Peterson, Solving the Ginzburg-Landau equations by finite element methods, Phys. Rev. B, 46 (1993), pp. 9027-9034.

[17] Y. ENomoto And R. Kato, The magnetization process in type II superconducting film, J. Phys. Condens. Matter, 4 (1992), pp. L433-L438.

[18] U. Essmann And H. TrÄUble, The direct observation of individual flux lines in type-II superconductors, Phys. Lett., A24, 10 (1967), pp. 526-527.

[19] J. Goodman, T. Y. Hou, And J. Lowengrub, Convergence of the point vortex method for the 2-d Euler equations, Comm. Pure Appl. Math., 43 (1990), pp. 415-430.

[20] T. Y. Hou And J. Lowengrub, Convergence of the point vortex method for the 3-d Euler equations, Comm. Pure Appl. Math., 43 (1990), pp. 965-981.

[21] B. J. Hunton, Vortex Dynamics, D. Phil. thesis, Oxford University, 1994.

[22] J. B. Keller, Propagation of a magnetic field into a superconductor, Phys. Rev., 111 (1958), p. 1497 .

[23] W. H. Kleiner, L. M. Roth, And S. H. Autler, Bulk solution of Ginzburg-Landau equations for type II superconductors: Upper critical field region, Phys. Rev. A, 133 (1964), p. 1226.

[24] E. Keryszig, Advanced Engineering Mathematics, John Wiley, New York, 1988.

[25] M. H. Millman And J. B. Keller, Perturbation theory of nonlinear boundary-value problems, J. Math. Phys., 10 (1969), p. 342.

[26] F. ODEH, Existence and bifurcation theorems for the Ginzburg-Landau equations, J. Math. Phys., 8 (1967), p. 2351.

[27] L. Peres and J. Rubinstein, Vortex motion in U(1) gauge models, Phys. D, 64 (1993), pp. 299-309.

[28] P. G. Saffman, Vortex Dynamics, Cambridge University Press, Cambridge, UK, 1993. 